DOI: https://dx.doi.org/10.26808/rs.ca.i8v1.21 International Journal of Computer Application (2250-1797)

Issue 8 Volume 1, January- February 2018

\title{
Bounded generalized Gaussian Mixture Model for Image Modeling
}

Osama Abdo Mohamed ${ }^{1,2}$, Omran Malik Omer Awad ${ }^{1}$ and Saif Elislam Adam Abdellah ${ }^{13}$

1 Department of Computer Science and Information Technology, Khulais Faculty of Information technology,Jeddah University, Saudi Arabia

2 Department of Mathematics, Faculty of Science, Zagazig University, Egypt

3 Department of Information Technology, Faculty of Computer Science and Information Technology, Alneelain University

\section{Abstract}

Many researchers studied the probability models for quantized and dequantized block Discrete Cosine transform(DCT) Coefficients in JPEG compression. However, All the studied probability models are unbounded. In many applications, the observed data are digitalized and have bounded support. A new bounded generalized Gaussian mixture model (BGGMM), which includes the Gaussian mixture model (GMM), Laplace mixture model(LMM), and GGMM(Generalized Gaussian Mixture Model) as special cases, is presented in this paper as a model for discrete cosine Coefficients.This new distribution has a flexibility to fit different shapes of observed data such as non-Gaussianand bounded support data. In order to estimate the model parameters, we propose an alternate approach to minimize the higher bound on the data negative log-likelihood function. Seven different probability distribution functions (Gaussian, Gamma, Rayleigh, Laplace, generalized Gamma, generalized Gaussian and bounded generalized Gaussian mixture) are compared for the accurate modeling of the DCT coefficients. We quantify the performance of the BGGMM with simulations and real data. It is shown via Kolmogorov-Smirnov (KS) tests that the PMFs based on the Bounded Generalized Gaussian Mixture Model (BGGMM) PDF are the most suitable distributions for DCT coefficients.

Keywords: BGGMM : Bounded Generalized Gaussian Mixture model ; GGM : Generalized Gaussian Model; GГ M: Generalized Gamma Model; GGMM:Generalized Gaussian Mixture Model, LMM: Laplace Mixture Model; GMM: Gaussian Mixture Model; K-S : Kolomogorve Simaranove test, DCT: Discrete Cosine Transform 
DOI: https://dx.doi.org/10.26808/rs.ca.i8v1.21 International Journal of Computer Application (2250-1797) Issue 8 Volume 1, January- February 2018

\section{.1. Introduction}

Due to the advances in various aspects of digital electronics like image acquisition, data storage and display, many new applications of the digital imaging have emerged over the past decade. However, many of these applications are not widespread because of required large storage space. As a result, the image compression has grown tremendously over the last decade and various image compression algorithms have been proposed. Joint Photographic Experts Group (JPEG) is a worldwide standard for the compression of still images.

Knowledge of the statistical distribution of the DCT coefficients is necessary in order to design the efficient quantizer with high SNR.Fig.1.b shows a typical plot of the histograms of the DCT coefficients. The image used here is the "man" picture shown in Fig. 1.a . The upper left coefficient is called the DC coefficient while the rest are AC coefficients. The scaling of the histogram is kept the same for all ac coefficients in this plot. It has empirically been proven that the DC coefficient, the coefficient at mode $(0,0)$ has a PDF that is different from the other modes of the DCT [9]. The AC coefficients, i.e., the coefficients at modes other than $(0,0)$ have been shown to have PDFs that are very similar to each other [3] [5] [9]. However, different papers have employed different PDFs to model AC block DCT coefficients. Early work on the distributions of 2D block DCT coefficients in images wascarried out by Pratt (1978) [8], who conjectured that the DC coefficients should beRayleigh distributed, since they are the sum of positive values (with the assumption of nolevelshift), and that the AC coefficients, in general, must be Gaussian distributed as perthe central limit theorem, with the assumption that each image pixel may be consideredas being statistically independent from another. Tescher (1979) [12] and Murakami,Hatori and Yamamoto (1982) [6] indicated that the AC coefficients are best modeled asLaplacian, and the DC coefficients are best modeled as Gaussian. Reininger and Gibson(1983) summarized the findings in their seminal paper [9] and concluded that the DCcoefficient is best modeled by a Gaussian PDF, while AC coefficients are best modeledby a Laplacian PDF. Reininger and Gibson used the KolmogorovSmirnov (KS) test [13] on standard256x256 8-bit PCM encoded grayscale images over multiple modes, to determine thegoodness of fit of the different PMFs.Muller [5] showed that the Generalized Gaussian distribution model (GG) fits the PDFof AC block DCT coefficients better than the Laplacian distribution using the $\chi 2$-Squaretest. In 2005, Chang, Shin, Kim and Mitra proposed a model for block DCT coefficients basedon the Generalized Gamma distribution (GrM). All the previously studied probability models are unbounded. In many applications, the observed data are digitalized and have bounded support. A new bounded generalized Gaussian mixture model (BGGMM), which includes the Gaussian mixture model (GMM), Laplace mixture 
DOI: https://dx.doi.org/10.26808/rs.ca.i8v1.21 International Journal of Computer Application (2250-1797)

Issue 8 Volume 1, January- February 2018

model(LMM), and GGMM(Generalized Gaussian Mixture Model) as special cases, is presented in this paper as a model for discrete cosine Coefficients. This new distribution has a flexibility to fit different shapes of observed data such as non-Gaussianand bounded support data. Seven different probability distribution functions (Gaussian, Gamma, Rayleigh, Laplace, generalized Gamma, generalized Gaussian and bounded generalized Gaussian mixture) are compared for the accurate modeling of the DCT coefficients.

The present paper has been divided as follows. In Section 2, Bounded Generalized Gaussian Mixture model is given. In Section 3, the parametric estimator of the presented statistical model is alsopresented. In section 4, we provide the experimental results of seven different distributions for four images and nine DCT coefficients. The last section concludes this paper.

\section{Bounded Generalized Gaussian Distribution Mixture (BGGMM)}

A Mixture of Bounded Generalized Gaussian Density [BGGMM] is a parametric statistical model which assumes that the data originates from weighted sum of several Bounded generalized Gaussian sources [11]. More specifically,

$$
g\left(x_{i} \mid \theta\right)=\sum_{j=1}^{K} m_{j} g_{j}\left(x_{i} \mid \mu_{j}, \sigma_{j}, \lambda_{j}\right)
$$

Where i $) \xi_{1}=\left(\mu_{1}, \mu_{2}, \ldots, \mu_{K}\right), \xi_{2}=\left(\sigma_{1}, \sigma_{2}, \ldots, \sigma_{K}\right), \xi_{3}=\left(\lambda_{1}, \lambda_{2}, \ldots, \lambda_{K}\right), \xi_{4}=\left(m_{1}, m_{2}, \ldots, m_{K}\right)$,

$$
=\bigcup_{i=1}^{4} \xi_{i}
$$

ii) $\mathrm{K}$ is the number of mixture density components,

iii) $m_{j}$ is the jth mixture weight and satisfies $m_{j} \geq 0, \sum_{j=1}^{K} m_{j}=1$

iv) $\mathrm{g}_{j}\left(x_{i} \mid \mu_{j}, \sigma_{j}, \lambda_{j}\right)$ is an individual density of the bounded generalized Gaussian density which introduced by Thanh Minh Nguyen, Q.M. Jonathan Wu and Hui Zhang [11 ] defined by

$$
\mathrm{g}_{j}\left(x_{i} \mid \mu_{j}, \sigma_{j}, \lambda_{j}\right)=\frac{\mathrm{T}_{j}\left(x_{i} \mid \mu_{j}, \sigma_{j}, \lambda_{j}\right) I\left(x_{i} \mid \Omega_{j}\right)}{\int_{\partial_{j}} \mathrm{~T}_{j}\left(x_{i} \mid \mu_{j}, \sigma_{j}, \lambda_{j}\right) I\left(x_{i} \mid \Omega_{j}\right) d x_{i}}
$$

The distribution $\mathrm{T}_{j}\left(x_{i} \mid \mu_{j}, \sigma_{j}, \lambda_{j}\right)$ in (3) is as follows:

$$
\mathrm{T}_{j}\left(x_{i} \mid \mu_{j}, \sigma_{j}, \lambda_{j}\right)=A\left(\lambda_{j}\right) \exp \left(-B\left(\lambda_{j}\right)\left|\frac{x_{i}-\mu_{j}}{\sigma_{j}}\right|^{\lambda_{j}}\right)
$$


Where

$$
A\left(\lambda_{j}\right)=\frac{\lambda_{j} \sqrt{\Gamma\left(3 / \lambda_{j}\right)}}{2 \sigma_{j} \Gamma\left(1 / \lambda_{j}\right) \sqrt{\Gamma\left(1 / \lambda_{j}\right)}} \text { and } \mathrm{B}\left(\lambda_{j}\right)=\left(\frac{\Gamma\left(3 / \lambda_{j}\right)}{\Gamma\left(1 / \lambda_{j}\right)}\right)^{\lambda_{j} / 2}
$$

The parameters $\mu_{j}$ and $\sigma_{j}$ are the mean and standard deviation. The parameter $\lambda_{j}$ controls the tails of the distribution and determines whether the latter is peaked or flat.

In (3), $\int_{\partial_{j}} \mathrm{~T}_{j}\left(x_{i} \mid \mu_{j}, \sigma_{j}, \lambda_{j}\right) I\left(x_{i} \mid \Omega_{j}\right) d x_{i}$ is the normalization constant and is identified as the share of $\mathrm{T}_{j}\left(x_{i} \mid \mu_{j}, \sigma_{j}, \lambda_{j}\right)$ that belongs to the supported region $\partial_{j}$ and $I\left(x_{i} \mid \Omega_{j}\right)$ is an indicator function defined for each component $\Omega_{j}$ that belongs to the supported region $\partial_{j}$ Where $I\left(x_{i} \mid \Omega_{j}\right)$ as:

$$
I\left(x_{i} \mid \Omega_{j}\right)= \begin{cases}1 & \text { if } x_{i} \in \partial_{j} \\ 0 & \text { otherwise }\end{cases}
$$

The importance of the bounded generalized Gaussian mixture model is that it has a flexibility to fit different shapes of observed data such as non-Gaussian and bounded support data and can be specialized to several important distributions Thanh Minh Nguyen, Q.M. Jonathan Wu and Hui Zhang [11] as shown in table 1

\begin{tabular}{|l|l|}
\hline GMM & BGGMM: $\lambda_{j}=\mathbf{2}, \boldsymbol{I}\left(\boldsymbol{x}_{\boldsymbol{i}} \mid \mathbf{\Omega}_{\boldsymbol{j}}\right)=\mathbf{1}$ \\
\hline LMM & BGGMM: $\lambda_{j}=1, I\left(x_{i} \mid \Omega_{j}\right)=1$ \\
\hline GGD & $K=1, I\left(x_{i} \mid \Omega_{j}\right)=1$ \\
\hline BGMM & BGGMM: $I\left(x_{i} \mid \Omega_{j}\right)=1$ \\
\hline BLMM & BGGMM: $\lambda_{j}=2, I\left(x_{i} \mid \Omega_{j}\right)=I\left(x_{i} \mid \Omega_{k}\right) ; \forall j, \quad k=(1,2, \ldots, \mathrm{K})$ \\
\hline
\end{tabular}

Table 1: some special distributions from the bounded generalized Gaussian mixture model(BGGMM)

Two important problems in the case of bounded generalized Gaussian mixture model are the estimation of the vector of parameters $\theta$ and the determination of the number of classes $\mathrm{K}$. When the number of classes is known, statistical inferential methods about the parameters can be used 
DOI: https://dx.doi.org/10.26808/rs.ca.i8v1.21 International Journal of Computer Application (2250-1797) Issue 8 Volume 1, January- February 2018

mostly via the Maximum-Likelihood estimation (MLE). In the Next section, we show how to estimate the model parameter and model order.

\section{Boundedgeneralized Gaussian mixturemodel Parameters Estimation}

Using the maximum likelihood method, the estimators of the unknown parameters vector $\theta=\left(\mu_{j}, \sigma_{j}, \lambda_{j}, \mathrm{~m}_{j}\right), j=1,2,3, \ldots \mathrm{K}$ can be obtained by taking the natural log of the likelihood function of a random sample of observation with the pdf in equation (1) then setting the derivatives of the log-likelihood function to zeros. The log-likelihood function of equation (1), given by [11] is

$$
\begin{aligned}
& E(\theta)=-\sum_{i=1}^{N} \sum_{j=1}^{K} z_{i, j}\left\{\log \mathrm{m}_{j}+\log \left(\mathrm{T}_{j}\left(x_{i} \mid \mu_{j}, \sigma_{j}, \lambda_{j}\right)\right)+\log \left(I\left(x_{i} \mid \Omega_{j}\right)\right)\right. \\
& \left.-\log \left(\int_{\partial_{j}} \mathrm{~T}_{j}\left(x_{i} \mid \mu_{j}, \sigma_{j}, \lambda_{j}\right) d x_{i}\right)\right\}
\end{aligned}
$$

where $\mathrm{N}$ the sample size and $z_{i j}$ is defined as [11]

$$
z_{i j}=\frac{m_{j} \psi\left(x_{i} \mid \mu_{j}, \sigma_{j}, \lambda_{j}\right)}{\sum_{l=1}^{K} m_{l} \psi\left(x_{i} \mid \mu_{l}, \sigma_{l}, \lambda_{l}\right)} \text { and } \psi\left(x_{i} \mid \mu_{j}, \sigma_{j}, \lambda_{j}\right)=\frac{\mathrm{T}_{j}\left(x_{i} \mid \mu_{j}, \sigma_{j}, \lambda_{j}\right) I\left(x_{i} \mid \Omega_{j}\right)}{\int_{\partial_{j}} \mathrm{~T}_{j}\left(x_{i} \mid \mu_{j}, \sigma_{j}, \lambda_{j}\right) d x_{i}}
$$

According to[11] the equations for estimating the parameters $\left(\mu_{j}, \sigma_{j}, \lambda_{j}, \mathrm{~m}_{j}\right)$ are [11]

$$
\frac{\partial E(\theta)}{\partial \mu_{j}}=-\beta\left(\lambda_{j}\right) \frac{\lambda_{j}}{\sigma_{j}^{\lambda_{j}}} \sum_{i=1}^{N} z_{i j}\left|x_{i}-\mu_{j}\right|^{\left(\lambda_{j}-2\right)}\left\{x_{i}+\mu_{j}-\frac{R_{j}}{\left|x_{i}-\mu_{j}\right|^{\left(\lambda_{j}-2\right)}}\right\}=0
$$

Where

$$
\begin{aligned}
& R_{j}=\frac{\sum_{m=1}^{M} \operatorname{sign}\left(\mu_{j}^{t}-S_{m j}\right)\left|\left(S_{m j}-\mu_{j}^{t}\right)\right|^{\left(\lambda_{j}(t)-1\right)} I\left(S_{m j} \mid \Omega_{j}\right)}{\sum_{m=1}^{m} I\left(S_{m j} \mid \Omega_{j}\right)} \\
& \frac{\partial E(\theta)}{\partial \sigma_{j}}=-\sigma_{j}^{-1} \sum_{i=1}^{N} z_{i j}\left\{-1+\beta\left(\lambda_{j}\right)\left|x_{i}-\mu_{j}\right|^{\lambda_{j}} \lambda_{j} \sigma_{j}^{-\lambda_{j}}-G_{j}\right\}=0
\end{aligned}
$$

Where 


$$
\begin{aligned}
& G_{j} \\
& =\frac{\sum_{m=1}^{M}\left\{-1+\lambda_{j}{ }^{(t)} \beta\left(\lambda_{j}{ }^{(t)}\right)\left|\left(S_{m j}-\mu_{j}^{(t)}\right)\right|^{\lambda_{j}{ }^{(t)}}\left(\sigma_{j}{ }^{(t)}\right)^{-\lambda_{j}(t)}\right\}}{\sum_{m=1}^{m} I\left(S_{m j} \mid \Omega_{j}\right)}
\end{aligned}
$$

To estimate the parameter $\lambda$ we use newton raphson method $[1,2,4]$ according to the following equation

$$
\lambda_{j}^{(t+1)}=\lambda_{j}^{(t)}-\frac{\partial E(\theta)}{\partial \lambda_{j}}\left(\frac{\partial^{2} E(\theta)}{\partial \lambda_{j}^{2}}+\gamma\right)
$$

Where $\gamma$ is a scaling factor. The derivatives of the error function with respect to $\lambda_{j}$ is given by

$$
\begin{aligned}
\frac{\partial E(\theta)}{\partial \lambda_{j}}=-\sum_{i=1}^{N} z_{i j}\left\{f\left(x_{i} \mid \mu_{j}, \sigma_{j}, \lambda_{j}\right)\right. & \\
- & \left.\frac{\int_{\partial_{j}} T_{j}\left(x \mid \mu_{j}, \sigma_{j}, \lambda_{j}\right) f\left(x \mid \mu_{j}, \sigma_{j}, \lambda_{j}\right) d x_{i}}{\int_{\partial_{j}} \mathrm{~T}_{j}\left(x_{i} \mid \mu_{j}, \sigma_{j}, \lambda_{j}\right) d x}\right\}
\end{aligned}
$$

Where $f\left(x \mid \mu_{j}, \sigma_{j}, \lambda_{j}\right)=$

$$
\begin{array}{r}
{\left[\frac{\frac{1}{\lambda_{j}}+3 \psi\left(\frac{1}{\lambda_{j}}\right)-3 \psi\left(\frac{3}{\lambda_{j}}\right)}{2 \lambda_{j}^{2}}\right]-\beta\left(\lambda_{j}\right)\left|\frac{x-\mu_{j}}{\sigma_{j}}\right|^{\lambda_{j}} \log \left(\left|\frac{x-\mu_{j}}{\sigma_{j}}\right|\right)-} \\
\quad \beta\left(\lambda_{j}\right)\left(\frac{1}{2}\right) \log \left(\frac{\Gamma\left(\frac{3}{\lambda_{j}}\right)}{\Gamma\left(\frac{1}{\lambda_{j}}\right)}\right)+
\end{array}
$$

$\psi 1 \lambda j-3 \psi 3 \lambda j 2 \lambda j x-\mu j \sigma j \lambda j$

$$
\begin{aligned}
\frac{\partial E(\theta)}{\partial^{2} \lambda_{j}}=-\sum_{i=1}^{N} z_{i j}\left\{g\left(x \mid \mu_{j}, \sigma_{j}, \lambda_{j}\right)-\frac{\left(\int_{\partial_{j}} T_{j}\left(x \mid \mu_{j}, \sigma_{j}, \lambda_{j}\right) f\left(x \mid \mu_{j}, \sigma_{j}, \lambda_{j}\right) d x_{i}\right)^{2}}{\left(\int_{\partial_{j}} \mathrm{~T}_{j}\left(x_{i} \mid \mu_{j}, \sigma_{j}, \lambda_{j}\right) d x\right)^{2}}\right. \\
\left.-\frac{\left(\int_{\partial_{j}} T_{j}\left(x \mid \mu_{j}, \sigma_{j}, \lambda_{j}\right)\left(f^{2}\left(x \mid \mu_{j}, \sigma_{j}, \lambda_{j}\right)+g\left(x \mid \mu_{j}, \sigma_{j}, \lambda_{j}\right)\right)\right) d x}{\int_{\partial_{j}} \mathrm{~T}_{j}\left(x_{i} \mid \mu_{j}, \sigma_{j}, \lambda_{j}\right) d x}\right\}
\end{aligned}
$$




$$
\begin{gathered}
\text { where } g\left(x \mid \mu_{j}, \sigma_{j}, \lambda_{j}\right)=\frac{\partial f\left(x \mid \mu_{j}, \sigma_{j}, \lambda_{j}\right)}{\partial \lambda_{j}}= \\
{\left[-\frac{1}{\lambda_{j}^{2}}-\frac{3 \dot{\psi}\left(\frac{1}{\lambda_{j}}\right)}{\lambda_{j}^{4}}-\frac{3 \psi\left(\frac{1}{\lambda_{j}}\right)}{\lambda_{j}^{3}}+\frac{9 \dot{\psi}\left(\frac{3}{\lambda_{j}}\right)}{2 \lambda_{j}^{4}}+\frac{3 \psi\left(\frac{3}{\lambda_{j}}\right)}{\lambda_{j}^{3}}\right]-} \\
\beta\left(\lambda_{j}\right)\left(\left(\frac{1}{2}\right) \log \left(\frac{\Gamma\left(\frac{3}{\lambda_{j}}\right)}{\Gamma\left(\frac{1}{\lambda_{j}}\right)}\right)+\frac{-3 \psi\left(\frac{3}{\lambda_{j}}\right)+\psi\left(\frac{1}{\lambda_{j}}\right)}{2 \lambda_{j}}+\frac{9 \dot{\psi}\left(\frac{3}{\lambda_{j}}\right)-\dot{\psi}\left(\frac{1}{\lambda_{j}}\right)}{2 \lambda_{j}^{3}}\right)\left|\frac{x-\mu_{j}}{\sigma_{j}}\right|^{\lambda_{j}} \log \left(\left|\frac{x-\mu_{j}}{\sigma_{j}}\right|\right)^{2}- \\
-3 \psi 3 \lambda j+\psi 1 \lambda j 2 \lambda j x-\mu j \sigma j \lambda \log x-\mu j \sigma j
\end{gathered}
$$

The term $\int_{\partial_{j}} \mathrm{~T}_{j}\left(x \mid \mu_{j}, \sigma_{j}, \lambda_{j}\right) d x$ in equations (8), (14) and (16) can be approximated as

$$
\int_{\partial_{j}} \mathrm{~T}_{j}\left(x \mid \mu_{j}, \sigma_{j}, \lambda_{j}\right) d x \approx \frac{1}{M} \sum_{m=1}^{M} I\left(S_{m j} \mid \Omega_{j}\right)
$$

Similarly, the term $\int_{\partial_{j}} T_{j}\left(x \mid \mu_{j}, \sigma_{j}, \lambda_{j}\right) f\left(x \mid \mu_{j}, \sigma_{j}, \lambda_{j}\right) d x_{i}$ in (14) and (16) can be approximated as :

$$
\int_{\partial_{j}} T_{j}\left(x \mid \mu_{j}, \sigma_{j}, \lambda_{j}\right) f\left(x \mid \mu_{j}, \sigma_{j}, \lambda_{j}\right) d x \approx \frac{1}{M} \sum_{m=1}^{M} f\left(S_{m j} \mid \mu_{j}^{(t)}, \sigma_{j}^{(t)}, \lambda_{j}^{(t)}\right) I\left(S_{m j} \mid \Omega_{j}\right)
$$

Similarly, the term $\left(\int_{\partial_{j}} T_{j}\left(x \mid \mu_{j}, \sigma_{j}, \lambda_{j}\right) f^{2}\left(x \mid \mu_{j}, \sigma_{j}, \lambda_{j}\right)+g\left(x \mid \mu_{j}, \sigma_{j}, \lambda_{j}\right)\right) d x$ in (16) can be approximated as :

$$
\begin{gathered}
\left(\int_{\partial_{j}} T_{j}\left(x \mid \mu_{j}, \sigma_{j}, \lambda_{j}\right)\left(f^{2}\left(x \mid \mu_{j}, \sigma_{j}, \lambda_{j}\right)+g\left(x \mid \mu_{j}, \sigma_{j}, \lambda_{j}\right)\right)\right) d x \\
\approx \frac{1}{M} \sum_{m=1}^{M}\left(f^{2}\left(x \mid \mu_{j}^{(t)}, \sigma_{j}^{(t)}, \lambda_{j}^{(t)}\right)\right. \\
\left.+g\left(x \mid \mu_{j}^{(t)}, \sigma_{j}^{(t)}, \lambda_{j}^{(t)}\right)\right) I\left(S_{m j} \mid \Omega_{j}\right)
\end{gathered}
$$

Solving equations (9) and (11) we get [11] 


$$
\begin{gathered}
\mu_{j}^{(t+1)}=\frac{\sum_{i=1}^{N} Z_{i j}\left(\left|x_{i}-\mu_{j}\right|^{\left(\lambda_{j}-2\right)} x_{i}+R_{j}\right)}{\sum_{i=1}^{N} Z_{i j}\left(\left|x_{i}-\mu_{j}\right|^{\left(\lambda_{j}-2\right)} x_{i}\right)} \\
\sigma_{j}^{(t+1)}=\frac{\lambda_{j} \mathrm{~B}\left(\lambda_{j}\right) \sum_{i=1}^{N} Z_{i j}\left|x_{i}-\mu_{j}\right|^{\lambda_{j}}}{\sum_{i=1}^{N} Z_{i j}\left(1+G_{j}\right)}
\end{gathered}
$$

To estimate the parameter $m_{j}$ which statisfy the constraint $m_{j} \geq 0, \sum_{j=1}^{K} m_{j}=1$ we use the following equation

$$
m_{j}^{(t+1)}=\frac{1}{N} \sum_{i=1}^{N} Z_{i j}^{(t)}
$$

\section{Experimental and results}

In this experiment the performance of Bounded Generalized Gaussian mixture Model (BGGMM) is compared to six statistical distribution functions ((Gaussian, Gamma, Rayleigh, Laplace, generalized Gamma, generalized Gaussian) for the accurate modeling of the DCT coefficients on a sample of thousand images.

K-S goodness of fit test statistic is used to measure the fitting accuracy of each distribution function.

The image histogram for DCT Coefficients for man image exist in figure 1.ais shown in figure1.b. Fitting the seven different distributions for $\mathrm{DC}, \mathrm{AC}(0,1), \mathrm{AC}(0,2)$ and $\mathrm{AC}(0,3)$ coefficients for image man are shown in figures 2. Figure 3 shows the mean KS test statistic values for nine discrete cosine coefficient blocks and seven statistical distribution functions of the studied images.

It can be seen from figure 2 and 3 that the Bounded Generalized Gaussian Mixture model KS statistic distance is smaller than all other KS statistic distance for the DCT coefficients. Therefore, it can be concluded that the distribution of the DCT coefficients (Both DC and AC) isBounded Generalized Gaussian Mixture in nature. Thus, Bounded Generalized Gaussian Mixture PDF can best approximate all of DCT coefficients for the studied images.

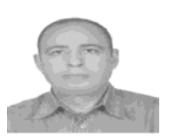


DOI: https://dx.doi.org/10.26808/rs.ca.i8v1.21 International Journal of Computer Application (2250-1797)

Issue 8 Volume 1, January- February 2018

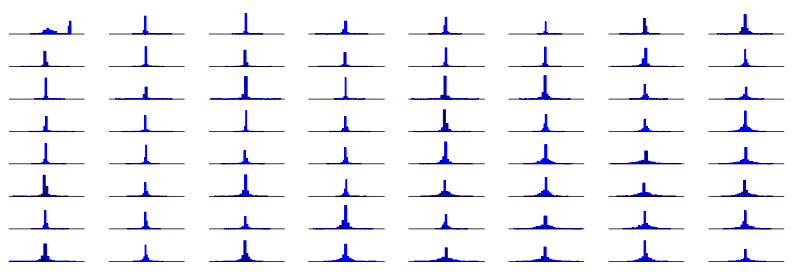

Figure: 1.b: Histogram of the DCT Coefficients for man image
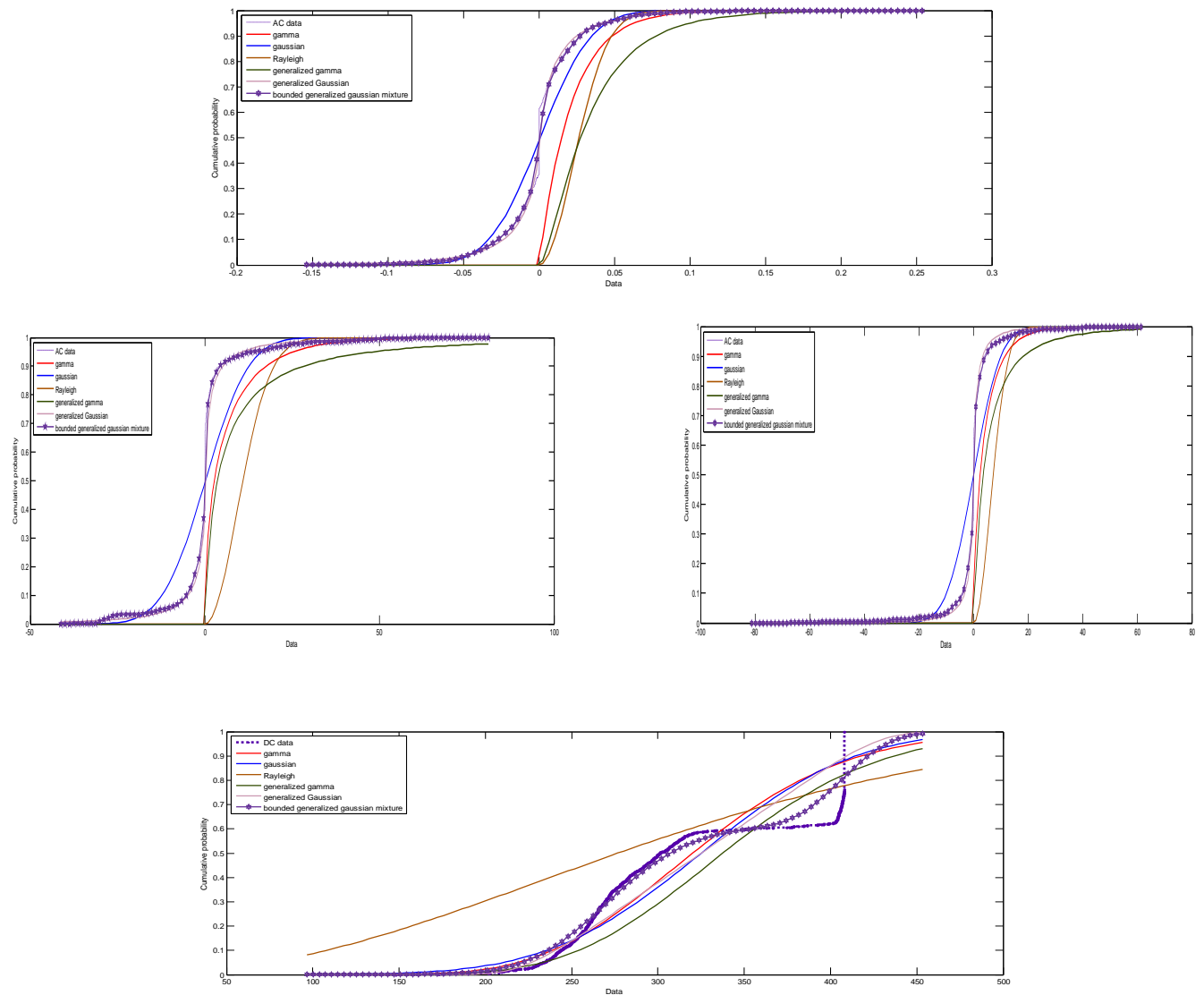

Figure: 2:fitting cumulative distributions for DC Cofficient for man image with gama, gaussain, Ray, GG,GГ and BGGM and laplace

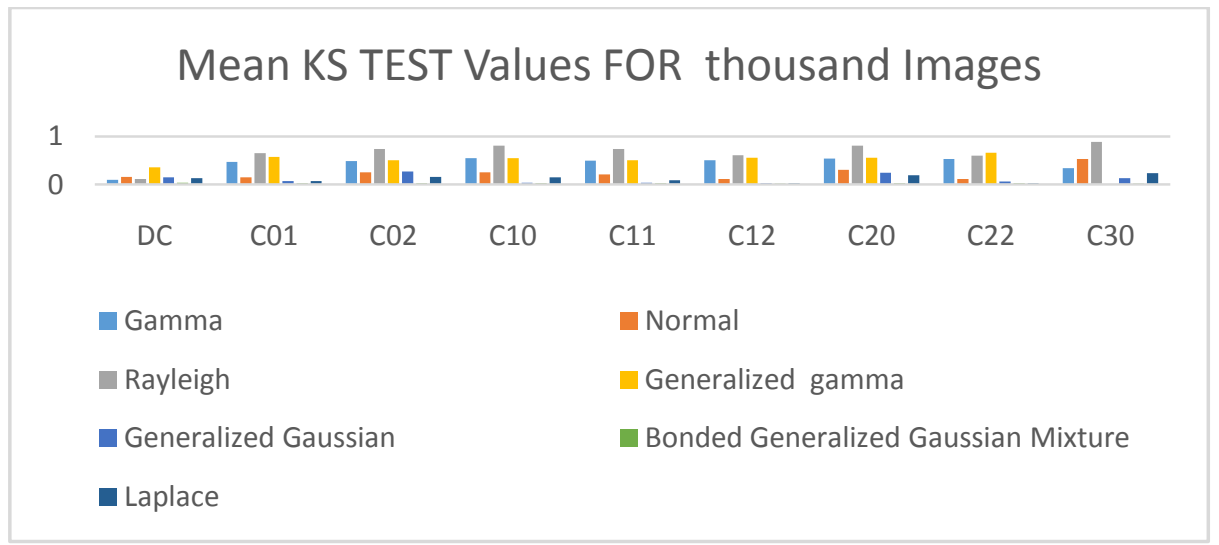

Figure 3: KS Statistics Results for seven probability distribution, for nine DCT coefficients, for thousand Images 
DOI: https://dx.doi.org/10.26808/rs.ca.i8v1.21 International Journal of Computer Application (2250-1797) Issue 8 Volume 1, January- February 2018

\section{Conclusions}

A new bounded generalized Gaussian mixture model (BGGMM), which includes the Gaussian mixture model (GMM), Laplace mixture model(LMM), and GGMM(Generalized Gaussian Mixture Model) as special cases, is presented in this paper as a model for discrete cosine coefficients.This new distribution has a flexibility to fit different shapes of observed data such as non-Gaussianand bounded support data. Seven different probability distribution functions (Gaussian, Gamma, Rayleigh, Laplace, generalized Gamma (GГ), generalized Gaussian (GG) and bounded generalized Gaussian mixture (BGGMM) ) are compared for the accurate modeling of the DCT coefficients. We quantify the performance of the BGGMM with simulations and real data. It is shown via Kolmogorov-Smirnov (KS) tests that the PMFs based on the Bounded Generalized Gaussian Mixture Model (BGGMM) PDF are the most suitable distributions for DCT coefficients.

\section{References}

[1] M. Allili, Wavelet modeling using finite mixtures of generalized Gaussian distributions: application to texture discrimination and retrieval, IEEETrans. Image Process.21(4)(2012)1452-1464.

[2] J. Ashburner, K.J.Friston,Unified segmentation,NeuroImage26(3)(2005) 839-851.

[3] J.-H. Chang, J. W. Shin, N. S. Kim, S. K. Mitra, Image Probability Distribution Based on Generalized Gamma Function, IEEE Signal Processing Letters, 2005.

[4] J. Lindblom, J. Samuelsson, Bounded support Gaussian mixture modeling of speech spectra,IEEETrans.SpeechAudioProcess.11(1)(2003)88-99.

[5] F. Muller, Distribution Shape of Two-Dimensional DCT Coefficients of Natural Images, Electronics Letters, 29, Oct. 1993, 1935-1936.

[6] H. Murakami, Y. Hatori, and H. Yamamoto, Comparison between DPCM and Hadamard transform coding in the composite coding of the NTSC color TV signal, IEEE Transactions on Communications, vol COM-30, pp. 469-479, Mar. 1982.

[7] S. Nadarajah, A generalized normal distribution, Journal of Applied Statistics.32(7), pp. 685-694, 2005.

[8] W. K. Pratt, Digital Image Processing, New York: Wiley-Interscience, 1978, chapter 10.

[9] R. Reininger and J. Gibson : Distribution of the two-dimensional DCT coefficients for images, IEEE Transactions on Communications 31 (6) 1983.

[10] E. W. Stacy, A Generalization of the Gamma Distribution, The Annals of Mathematical Statistics. Vol. 33, No. 3, pp. 1187-1192, September, 1962.

[11] Thanh Minh Nguyen, Q. M. Jonathan Wu, Hui Zhang, Bounded generalized Gaussian mixture model, Journal of Pattern Recognition. Vol 47, pp. 3132-3142, (2014) 
DOI: https://dx.doi.org/10.26808/rs.ca.i8v1.21 International Journal of Computer Application (2250-1797) Issue 8 Volume 1, January- February 2018

[12] A. G. Tescher, Transform image coding, in Advances in Electronics and Electron Physics. Suppl. 12. New York: Academic, 1979, pp. 113-115.

[13] E. W. Weisstein, Kolmogorov-Smirnov Test. From Math World--A Wolfram Web Resource. http://mathworld.wolfram.com/Kolmogorov-SmimovTest.html, retrieved March 22, 2010. 